\title{
Cognitive Behavioural Therapy for Japanese Children and Adolescents with Anxiety Disorders: A Pilot Study
}

\author{
Shin-ichi Ishikawa \\ Doshisha University, Kyoto, Japan \\ Naoyasu Motomura, Yasuo Kawabata and Hidetaka Tanaka \\ Osaka Medical College, Japan \\ Sakie Shimotsu \\ Ryukoku University, Kyoto, Japan \\ Yoko Sato \\ University of Miyazaki, Japan \\ Thomas H. Ollendick \\ Child Study Center, Virginia Tech, Blacksburg, USA

\begin{abstract}
Background: Thirty-three Japanese children and adolescents diagnosed with an anxiety disorder participated in individual or group Cognitive Behaviour Therapy (CBT) that was modelled after evidence-based intervention programs developed in Western countries. Method: The treatment consisted of: (a) building rapport and education; (b) identifying emotions and recognizing cognitive self-talk; (c) challenging anxious self-talk; (d) developing an anxiety hierarchy and in vivo exposures; and (e) planning for future challenges. Results: Three months following treatment, 20 of the 33 children and adolescents $(60.91 \%)$ no longer met criteria for their principal anxiety disorders and $16(48.48 \%)$ were free from all anxiety disorders. Self-reported anxiety, depression, and cognitive errors also decreased significantly from pre- to post-treatment and these gains were maintained at 3-month follow-up. For the most part, similar outcomes were found in both the group and individual formats of CBT.
\end{abstract}

Reprint requests to Shin-ichi Ishikawa, Faculty of Psychology, Doshisha University, Imadegawa-dori, Karasuma, Higashi-iru, Kamigyo-ku, Kyoto, Japan 602-8580. E-mail: ishinn@mail.doshisha.ac.jp 
Conclusions: This study provides preliminary support for the transportability of CBT in both an individual and group format to Japan.

Keywords: Cognitive behavioural therapy (CBT), children, adolescents, anxiety disorders.

\section{Introduction}

Anxiety disorders are common in children and adolescents (Albano, Chorpita and Barlow, 2003; Ollendick and March, 2004). Prevalence studies indicate 3-month prevalence rates of $2.9 \%-8.6 \%$, 6-month prevalence rates of 9.1\%-17.7\%, and lifetime prevalence rates of 12.7\%-27.0\% by young adulthood (Costello, Egger and Angold, 2004). Anxiety disorders are also known to cause functional impairments in school, home, and community settings (e.g. Benjamin, Costello, and Warren, 1990; Ollendick and King, 1994). In addition, fear and anxiety in childhood predict later psychiatric disorders, including other anxiety disorders, depression, and substance use (Kendall, Safford, Flannery-Schroeder and Webb, 2004; Pine, Cohen, Gurley, Brook and Ma, 1998; Rohde, Lewinsohn and Seeley, 1991, 1996).

Several randomized controlled trials have supported the efficacy of cognitive behaviour therapy (CBT) in group and individual formats for anxiety and phobic disorders in children and adolescents. Reviews of the literature suggest that CBT meets criteria for a "probably efficacious treatment" in as much as it has been shown to be superior to waitlist controls in these studies (Ollendick and King, 1998; Silverman, Pina and Viswesvaran, 2008). Since the recent review by Silverman and colleagues, additional randomized controlled trials with a large number of participants have been conducted that now suggest CBT is a treatment that is "well-established". That is, it has been shown to be superior to attention control and other treatments. For example, Ollendick and colleagues (2009) conducted a randomized controlled trial of one-session exposure treatment for specific phobias. Participants included 196 youth between 7 and 16 years of age from Sweden and the United States. One session exposure treatment outperformed education support treatment as well as a waitlist condition in this study. Similarly, Beidel and colleagues (2007) evaluated the efficacy of fluoxetine, pill placebo, and Social Effectiveness Therapy for Children (SET-C). In this study, 122 children and adolescents (ages 7 to 17) from the US with social phobia participated. Although both pharmacologic and psychosocial treatments were more efficacious than pill placebo, SET-C was superior to fluoxetine regarding social distress, behavioural avoidance, and general functioning.

Finally, in the largest trial ever undertaken, the Child-Adolescent Anxiety Multimodal (CAMS) Study was designed as a multi-site randomized controlled trial for 488 children and adolescents between the ages of 7 and 17 years who had separation anxiety disorder, generalized anxiety disorder, or social phobia (Walkup et al., 2008). This study, also conducted in the US, included two phases. Phase 1 was a 12-week trial for comparing four treatment conditions: cognitive behavioural therapy, sertraline, a combination of these two treatments, and a placebo drug. Phase 2 was a 6-month open-extension. Although findings were mixed, results of clinician rating scales showed that all three treatments were superior to pill placebo; in addition, the combination therapy was superior to both mono-therapies.

Despite these promising results of CBT, these trials have all been conducted in Western countries with similar cultural values (Essau, Sakano, Ishikawa and Sasagawa, 2004). Unfortunately, few clinical trials with children and adolescents from other cultural backgrounds have been conducted. In order to disseminate evidence-based treatments, it is 
critical to conduct clinical trials for other races, ethnicities, and cultures. Regarding cultural differences, Markus and Kitayama (1991) identified two types of self-construal that are culture-dependent: interdependent and independent self-construals. According to Markus and Kitayama (a) individuals with independent self-construals (common in Western countries) tend to emphasize individualistic aspects of the self, and (b) people with interdependent self-construals (common in Asian countries such as Japan) tend to emphasize collectivistic aspects of the self. For instance, while children with interdependent self-construals emphasize the maintenance of harmony within their group and tend to avoid arguments and conflicts, children with independent self-construals prefer to be more assertive and even to argue if necessary to get their ideas across or to get their way in social interactions. On the basis of this distinction, it is important to determine whether group or individual CBT works differently in youth from interdependent, collectivistic culture. As noted above, both group and individual CBT have been shown to be equally effective with children from Western countries who tend to possess independent self-construals.

On the surface, interdependent self-construals and overall collectivism might seem to work against the efficacy of group CBT in Japan; this is so because accomplishment of individual goals such as improvement in anxiety symptoms is not emphasized, at least not in comparison to maintenance of harmony within the group. However, group CBT for patients with anxiety symptoms conducted in Japan had been shown to be not only feasible but also highly effective. For example, Chen and colleagues (2007) demonstrated the effectiveness of a group CBT program based on an evidence-based program in Western countries for Japanese adult patients with social anxiety disorders. For the Intent to Treat (ITT) analyses, the percentage of reduction was $20 \%$ to $30 \%$ in diagnostic status and pre to post effect size ranged from medium to large, $0.37-1.01$. In addition, they reported that most of the patients appeared to be comfortable with this treatment format.

Similarly, Shimotsu and colleagues evaluated the effectiveness of group CBT for anxious and depressed patients. At both posttreatment and 3-month follow-up, significant improvements were found in anxiety symptoms, depressive symptoms, and overall maladaptive belief (Shimotsu et al., 2011). Furthermore, quality of life in physical, psychological, social, and environmental aspects significantly improved at the 3-month follow-up. Based on these pilot trials with adults, it is reasonable to assume that group CBT would also be acceptable for Japanese children with anxiety disorders. Moreover, the group format might be more familiar and common for school-aged children and adolescents in Japan. In fact, previous meta-analysis (Ishikawa, Okajima, Matsuoka and Sakano, 2007) suggested the efficacy of group format treatment for children and adolescents. More specifically, younger age groups experienced the largest improvements when cohesion was present in the group (Burlingame, McClendon and Alonso, 2011). Therefore, group CBT in Japan might be as effective as individual CBT, assuming the group members share individual goals and work together in small cohesive groups.

In the present paper, the focus was on CBT for children with anxiety disorders in Japan and included an initial comparison between group and individual formats. Anxiety problems appear to be as common in Japan as they are in Western countries. In an earlier study, Ishikawa, Sato and Sasagawa (2009) investigated anxiety symptoms in Japanese children and adolescents using the Spence Children's Anxiety Scale (SCAS; Spence, 1998). Mean score on the SCAS of Japanese children and adolescents were similar to those found in Western countries (see Ishikawa et al., 2009). Thus, anxiety symptoms appear not only to 
be present in Japanese youth but also to be comparable to those found in Western countries. However, there have been no studies to date of CBT for children and adolescents with anxiety disorders in Japan, or for that matter other non-English speaking countries in Asia; to our knowledge, this is the first study to do so. There were three hypotheses in the present study. First, it was expected that CBT would provide similar therapeutic effects for Japanese children and adolescents with anxiety disorders in line with previous studies in Western countries. Second, based on previous findings in Western countries, it was hypothesized that group CBT would be comparable to individual CBT in terms of youth who were diagnosis-free following treatment and on the measures of anxiety, depression, and cognitive errors. Third, as previously suggested (see Kendall and Treadwell, 2007; Treadwell and Kendall, 1996), we predicted that changes in cognitive errors would be related to improvement of anxiety and depression.

\section{Method}

\section{Participants}

Children and adolescents were recruited through flyers at schools, community clinics, university clinics, and newspaper advertisement in Osaka and Miyazaki, Japan. Osaka is the second largest city in Japan and located in the middle part of Honshu. Miyazaki is located in the Kyushu district on the south main island in Japan. Both are considered to be progressive cities and largely middle class in nature. To be included in the study the participants (a) had to be between 7 and 15 years of age, (b) have an anxiety disorder as determined through a diagnostic interview (see below), (c) not fulfill criteria for PTSD, mental retardation, pervasive developmental disorder, or a psychotic disorder, and (d) agree to discontinue other forms of psychotherapy for the duration of the study.

Through the referral process, 41 potential participants were evaluated between 2006 and 2010; however, 8 youth did not meet inclusion criteria: 6 children fulfilled criteria for mental retardation or pervasive developmental disorder and 2 did not have an anxiety disorder. Thirtythree children and adolescents met inclusion criteria: 20 girls (60.60\%) and 13 boys (39.39\%), with a mean age of 11.24 years $(S D=2.22)$. Sixteen youth met criteria for social phobia, 8 for generalized anxiety disorder, 4 for separation anxiety disorder, and 5 for specific phobia. Twenty-two of the 33 participants $(66.67 \%)$ had more than one anxiety disorder and 11 $(33.33 \%)$ had three or more anxiety disorders. In addition, 6 youth had dysthymic disorder and 1 child had selective mutism. All participants were Japanese in ethnicity and lived with both parents. No participants were taking anti-anxiety or antidepressant medications during the study. In accordance with IRB requirements in our country, information about SES could not be collected; however, most participants were from geographic areas that are considered to be middle class in socioeconomic status.

\section{Measures}

Diagnostic interview. The Anxiety Disorders Interview Schedule for DSM-IV (ADIS; Silverman and Albano, 1996) was administered by trained clinical psychologists. The same clinician interviewed the child and his/her parents; diagnoses were based on information provided by both informants (i.e. composite diagnoses). The ADIS was translated to Japanese for the purpose of this study. One bilingual translator translated the questionnaire from English 
to Japanese, and another bilingual translator who was also a native speaker back-translated the interview into English. Differences in the two versions were discussed and resolved by joint agreement of both translators.

Child-self report: The Spence Children's Anxiety Scale (SCAS; Spence, 1998). The SCAS is a 38-item measure of anxiety symptoms for children and adolescents from 8 to 15 years of age. Ishikawa et al. (2009) confirmed the validity and reliability of the translated version of SCAS. The SCAS has six factors: separation anxiety disorder; social phobia; obsessivecompulsive disorder; panic attack and agoraphobia; physical injury fear; and generalized anxiety disorder. Each item is rated on a 4-point scale in terms of its frequency from $0=$ never to 3 = always. The Cronbach's alpha for the SCAS was .82 in the present study.

Depression Self-Rating Scale (DSRS; Birleson, 1981). The DSRS is an 18-item measure of depressive symptoms for children and adolescents between 6 and 15 years of age. Murata, Shimizu, Mori and Oushima (1996) translated the English scale into Japanese. The DSRS has a single factor structure and each item is rated on a 3-point scale in terms of its frequency from $0=$ never to $2=$ always. The Cronbach's alpha for the DSRS was .80 in the present sample.

Children's Cognitive Error Scale (CCES; Ishikawa and Sakano, 2003; Ishikawa, in press). The CCES is 20-item measure for assessing children's cognitive errors. The CCES consisted of two parts: a series of hypothetical scenarios and the interpretation of those scenarios. For example, one hypothetical scenario was: "Yesterday after school, you played with your friend longer than usual. Today, your friend was absent from school even though he/she was fine yesterday." Possible interpretations of this situation could be: "My friend may be absent tomorrow, as well," or "It is my fault that my friend is absent because we played longer than usual." The hypothetical scenarios in each vignette were collected using an openended question method with Japanese children, and its content and socio-ecological validity were confirmed. Each possible interpretation in the CCES was designed to assess four major cognitive errors (catastrophizing, overgeneralizing, personalizing, and selective abstraction) based on the previous work of Leitenberg, Yost and Carroll-Wilson (1986). Previous studies have shown sufficient validity and reliability of the CCES for assessing cognitive errors in Japanese youth (Ishikawa and Sakano, 2003; Sato, Ishikawa and Arai, 2004).

A multi-group confirmatory factor analysis in another study supported the single factor structure of the CCES, $\chi^{2}=664.24, d f=341, \mathrm{GFI}=.98, \mathrm{AGFI}=.98, \mathrm{NFI}=.97, \mathrm{RFI}=.97$ and PNFI $=.87$ (Ishikawa, in press). The reliability coefficients were calculated separately for children in elementary school from 8 to 12 years of age and adolescents who attended junior high school and were between 12 and 15 years of age. Alpha coefficients were .92 and .89 and mean inter-item correlation was .62 and .57 , respectively. Test-retest reliabilities in children and adolescents were significant, ICC $=.69, p<001$ and ICC $=.81, p<001$ (children and adolescents, respectively).

Each item is rated on a 4-point scale in terms of its frequency from $0=$ never think so to $3=$ think so very much. In the present study, the CCES possessed high internal consistency, with a Cronbach's alpha of .98 .

\section{Procedure}

Upon receipt of a referral, a member of the research team conducted a brief telephone screening interview. Parents were asked about children's primary problems and the presence 
Table 1. Program components

\begin{tabular}{ll}
\hline No. & \multicolumn{1}{c}{ Contents } \\
\hline 1 & Build rapport and provide education \\
2 & Identification of emotions \\
3 & Introduce the relationship between thoughts and emotions \\
4 & Recognition and identification of anxious self-talk \\
5 & Challenging anxious self-talk \\
6 & Designing an anxiety staircase \\
7 & In vivo exposure \\
8 & Review the program and planning for future challenges \\
\hline
\end{tabular}

Note: Cognitive Behaviour Therapy Program for Japanese children and adolescents with anxiety disorders (Ishikawa, Shimotsu and Sato, 2008)

of any disorders meeting exclusionary criteria. If a child was considered a potential participant, an appointment was set up for the pretreatment assessment. The interview with the child and the parents (frequently the mother only) included the purpose of the study, an explanation of CBT, the diagnostic interview, the self-report measures, and the completion of informed consent/assent forms. Only children and parents who signed an informed consent/assent form participated in this study. If the child was judged to fulfill any anxiety disorders of DSM-IV criteria from the ADIS, he or she continued filling out the SCAS, DSRS, and CCES questionnaires.

A quasi-experimental design was used such that youth were assigned to either a group or individual format, but not in a randomized manner. Twenty (4 from Osaka and 16 from Miyazaki; 8 boys and 12 girls; mean age $=11.05$ years of age, $S D=2.24$ ) children and adolescents were assigned to the group format and 13 (8 from Osaka and 5 from Miyazaki; 5 boys and 8 girls; mean age $=11.54$ years of age, $S D=2.33$ ) were assigned to the individual treatment format. Youth were disproportionately assigned in the two cities due to the availability of therapists there and the recruitment of participants into the study. Although they were assigned non-randomly to the CBT format, there were no significant differences between participants in the individual and group format conditions regarding gender, mean age, number of diagnoses, and self-report measures before treatment.

Participants were provided with eight 90-minute-sessions of treatment. Upon completion of treatment, participants and their parents were invited back for a posttreatment assessment interview. During this posttreatment assessment session, the ADIS was re-administered to both children and parents and the three self-report questionnaires were re-administered. These procedures were also followed at 3-month follow-up.

\section{Cognitive behaviour therapy}

The therapists followed the CBT Program for Japanese children and adolescents with anxiety disorders developed by Ishikawa, Shimotsu and Sato (2008). This program was based on previous evidenced-based programs (e.g. Barrett, 1998; Kendall, 1994) and consisted of: (a) building rapport and education; (b) identifying emotions and cognitive self-talk; (c) challenging anxious self-talk; (d) developing an anxiety hierarchy and graduated in vivo exposures; and (e) planning for the future (see Table 1). As noted, eight 90-minute sessions 
were conducted. Following the eight sessions, three booster sessions were provided once per month to review progress of in vivo exposures during their daily lives. Six licensed Japanese clinical psychologists provided treatment. Each therapist participated in a 2-day training session delivered by the first author. Ishikawa has conducted CBT with children and adolescents for 10 years and has received training in CBT at the Association of Behavioural and Cognitive Therapies and the World Conference on Cognitive and Behavioural Therapies.

Group cognitive behaviour therapy. Group CBT was facilitated by licensed clinical psychologists in both cities who attended the above training sessions. Other clinical psychologists and graduate students participated as co-facilitators. Each group consisted of 3 - 6 children and adolescents. Group assignment was determined by the principal investigators of each site (SI and NM) based on the participants' ages, gender, and principal diagnoses. Although there were no structured parental sessions per se, parents were invited to observe sessions from the back of the therapy room and to participate in sessions when invited to do so (e.g. planning homework exposure or establishing reinforcement programs).

Individual cognitive behaviour therapy. Individual CBT was also delivered by clinical psychologists in both cities who attended the above training sessions. Parents were in the same room as the child, received the explanation of treatment, and managed homework tasks as needed. In as much as possible, the involvement of the parents was the same in both groups. The primary difference between the two conditions is that only one child and his or her parents attended the therapy session in the individual format whereas up to six children and their parents attended the sessions in the group format.

\section{Results}

After initial assignment to treatment, one boy and one girl (both 13 years of age) did not complete the treatment. Both of these individuals were in the group intervention format in Miyazaki. The main reason for their discontinuation was reported to be difficulty with arranging their family schedules for participation. As a result, 31 children and adolescents (93.94\%; 19 girls and 12 boys; $M=11.13$ years old, $S D=2.24$ ) completed treatment. Both completer and intent-to-treat (ITT) analyses are reported. ITT analyses were conducted using the last observation carried forward (LOCF) method.

\section{Diagnostic interview}

According to diagnostic interviews, 18 children (54.55\% for ITT, 58.06\% for completers) were free of their primary anxiety disorder following treatment. Furthermore, 20 children (60.61\% for ITT, $64.52 \%$ for completers) were free from their principal anxiety disorder at the 3-month follow-up. Twelve children (36.36\% for ITT, $38.71 \%$ for completers) at posttreatment and 16 children ( $48.48 \%$ for ITT, $51.61 \%$ for completers) at 3 months were free of all anxiety disorders. In terms of diagnostic free rates, there were no significant differences between individual and group formats at post-treatment, $\chi^{2}(1)=1.87$ for principal and $\chi^{2}(1)=3.22$ for all diagnoses. Similarly, we did not find any significant differences at 3 -month follow-up, $\chi^{2}(1)=0.53$ and $\chi^{2}(1)=0.49$, for principal and all diagnoses, respectively. 


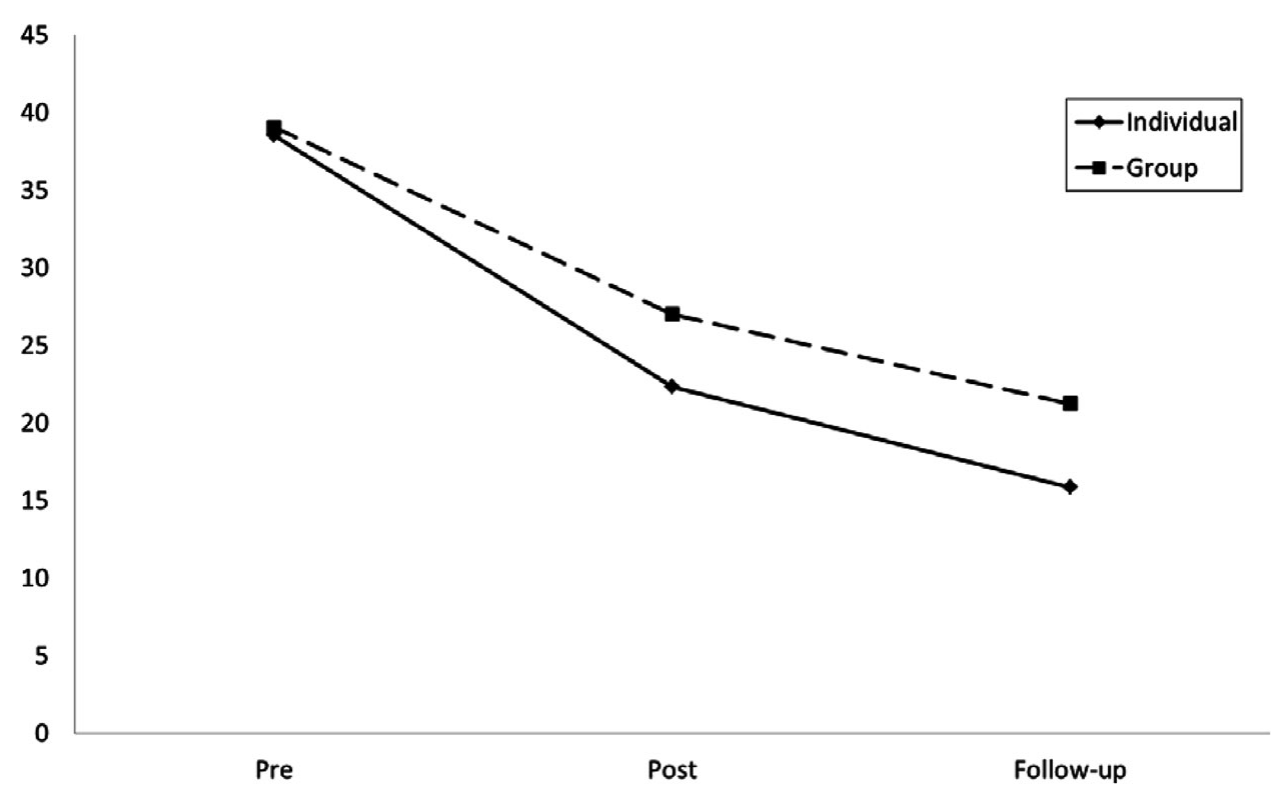

Figure 1. Pre-, post-, and follow-up anxiety scores for individual and group cognitive behavior therapy

\section{Self-report measures}

In order to evaluate the effects of CBT on self-reports of anxiety, depression, and cognitive errors, a mixed model analysis of variance was used. Time (pre, post, and 3-month followup), format (individual vs. group), and the interaction term were included as fixed effects and participants were viewed as random effects. We completed these analyses both for the completers and the ITT sample. For the completers, results revealed significant time effect for anxiety symptoms, $F(2,57.33)=30.32, p<.001$. Multiple comparison using Bonferroni's method indicated anxiety symptoms decreased significantly from pre- to posttreatment and these effects were maintained at the 3-month follow-up, $p<.001$ (Figure 1). Format and interaction effects were not significant. Depression also showed a significant time effect, $F(2,56.81)=31.85, p<.001$. Multiple comparisons demonstrated that depression not only decreased significantly at posttreatment but decreased even further at the time of the 3 -month follow-up, $p<.001$ and $p<.05$, respectively (Figure 2). Although the format effect was not significant, the interaction of time and format was significant, $F(2,56.81)=$ $6.38, p<.01$. Simple main effect tests revealed that children who participated in individual treatment showed significant improvement from pre- to posttreatment and to 3-month followup, $p<.001$, whereas children in the group format did not change from pre- to posttreatment. They did, however, change from pretreatment to follow-up, $p<.001$. For cognitive errors, a significant time effect was also found, $F(2,57.22)=26.29, p<.001$. Multiple comparison indicated cognitive errors improved significantly at posttreatment and these effects were maintained at the 3-month follow-up, $p<.001$ (Figure 3). Format and interaction effects were not significant. 


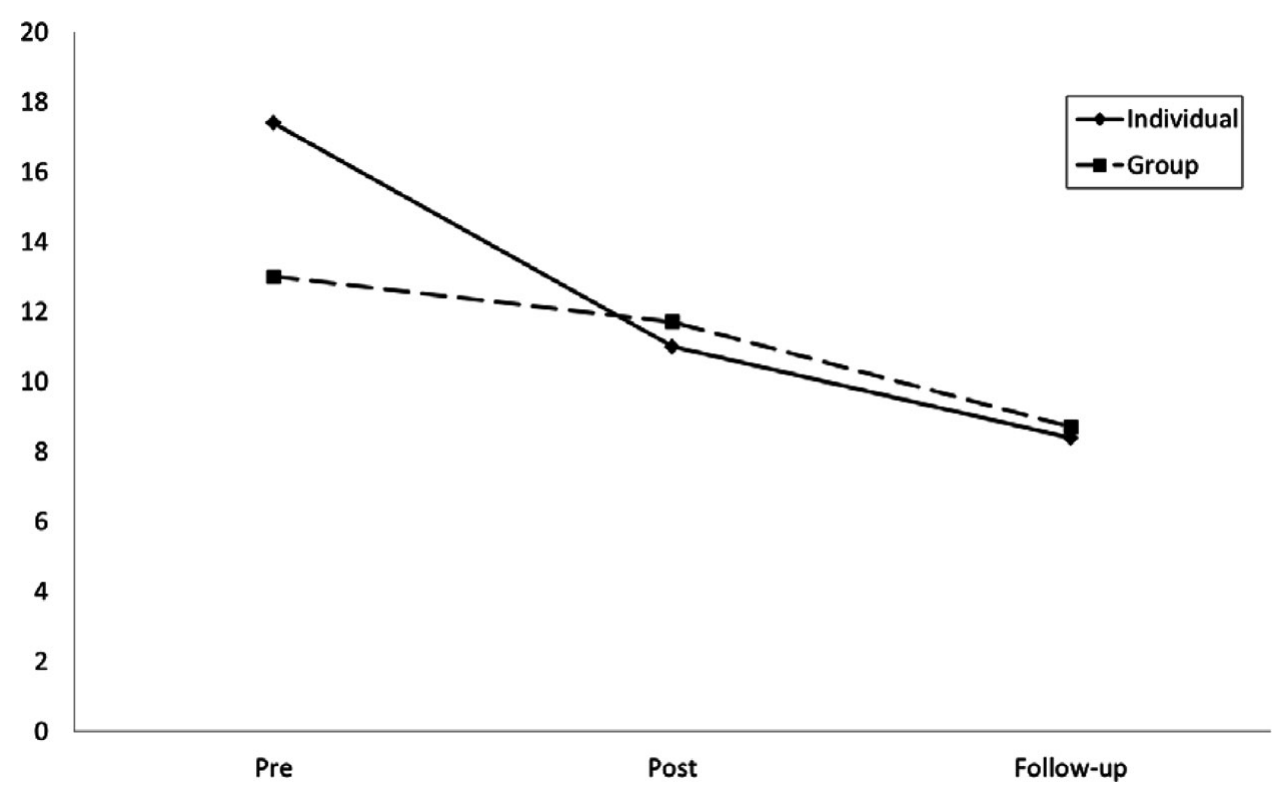

Figure 2. Pre-, post-, and follow-up depression scores for individual and group cognitive behavior therapy

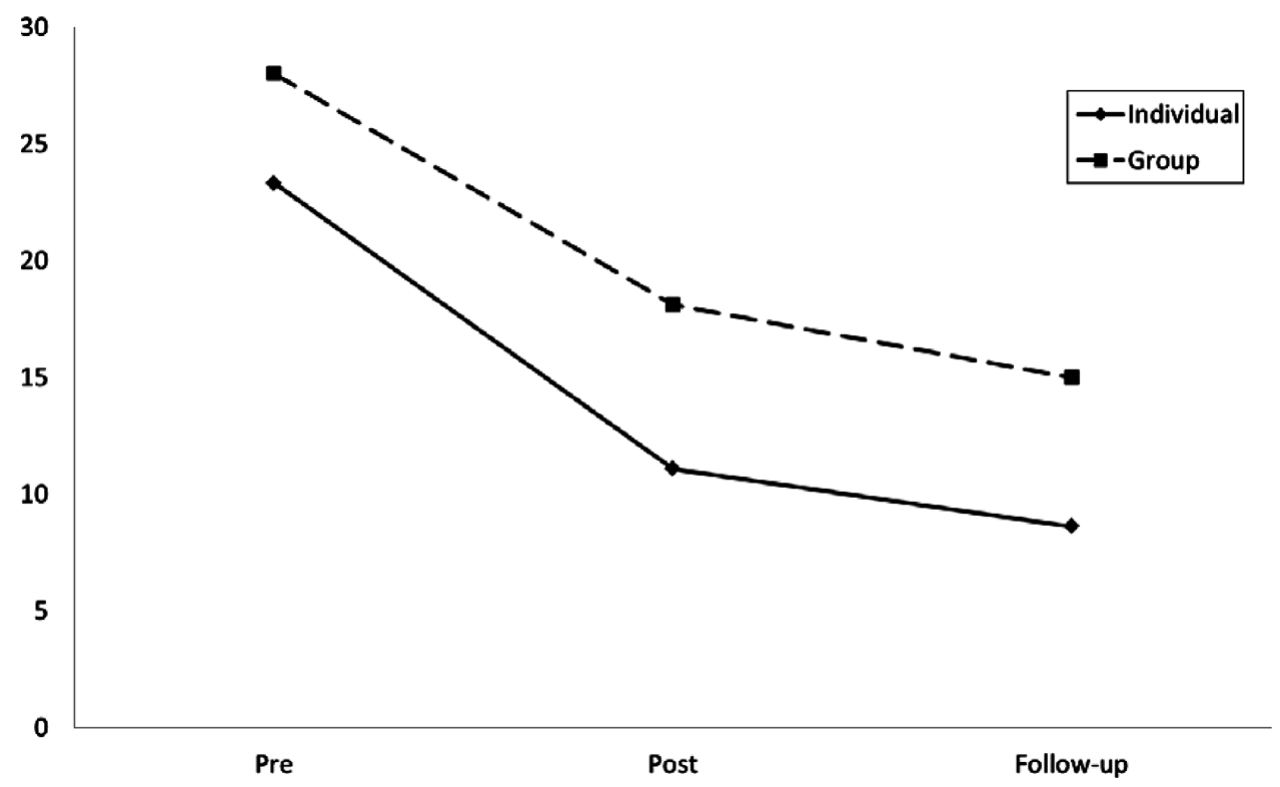

Figure 3. Pre-, post-, and follow-up cognitive errors scores for individual and group cognitive behavior therapy 
Table 2. Correlation of change scores

\begin{tabular}{llllll}
\hline & \multicolumn{2}{c}{ Pre to post } & & \multicolumn{2}{c}{ Pre to follow-up } \\
\cline { 2 - 3 } \cline { 6 - 7 } & SCAS & DSRS & & SCAS & DSRS \\
\hline CCES (pre-post) & $0.32^{\dagger}$ & 0.17 & & $0.48^{* *}$ & $0.39^{*}$ \\
CCES (pre-fu) & $0.40^{*}$ & 0.10 & & $0.51^{* *}$ & $0.38^{*}$ \\
\hline
\end{tabular}

Note CCES $=$ Children's Cognitive Error Scale, DSRS

$=$ Depression Self-Rating Scale, SCAS = Spence

Children's Anxiety Scale

${ }^{\dagger} p<.10,{ }^{*} p<.05,{ }^{* *} p<.01$

We conducted similar analyses including the two adolescents who did not complete this program (ITT) for the three self-report measures. Result also indicated significant time effect for the SCAS, DSRS, and CCES, $F(2,61.79)=31.06, F(2,61.07)=34.19, F(2,61.39)=$ $26.13, p<.001$, respectively. Time and format interaction effect was also significant for the depression measure, $F(2,61.07)=34.19, p<.01$. Simple main effect tests supported the same transitions for both individual and group treatments to completer analyses. Furthermore, a format main effect was significant for the CCES, $F(2,29.72)=4.47, p<.05$. Since both adolescents who did not complete the treatment were in the group format and their pretreatment scores were carried forward, cognitive errors in group format were higher than those in the individual treatment.

Finally, in order to evaluate the effect size associated with our main findings, within-subject $d$ values were calculated for completers. The effect sizes of anxiety symptoms were $d=1.28$ for pre-post and $d=1.56$ for pre-follow-up. For depression, effect sizes were $d=0.85$ for pre-post and $d=1.45$ for pre-follow-up. For cognitive errors, effect sizes were $d=1.24$ for pre-post and $d=1.51$ for pre-follow-up.

\section{Relationship between cognitive change and improvements in anxiety and depression}

We also examined the relationship between changes in cognitive errors and improvement in anxiety and depressive symptoms. Table 2 shows the results of Pearson correlation analyses for the change scores on each measure. Cognitive change from pre- to posttreatment correlated significantly to improvement in both anxiety and depression from pre to follow-up, but not from pre- to posttreatment.

\section{Discussion}

The present study evaluated CBT for Japanese children and adolescents with anxiety disorders. More than half of the youth who participated in CBT were free from their principal anxiety disorder diagnosis following eight sessions of treatment. At the 3-month followup, 20 of the 31 children and adolescent $(60.91 \%)$ who attended CBT were without their primary anxiety disorder. Furthermore, approximately half of the participants (48.48\%) no longer fulfilled criteria for any anxiety disorder. Consistent with results from the diagnostic interview, children showed significant improvements in self-report anxiety and depression, as well as changes in the extent of their cognitive errors. Moreover, these effects were found 
regardless of treatment format except for depression in group CBT. To our knowledge, the present study is the first trial in Japan in terms of CBT for children and adolescents with anxiety disorders.

Recovery rates observed in this study were consistent with previous randomized controlled trials conducted in Western countries. Silverman and colleagues showed that $63 \%$ of youth were diagnosis-free from any anxiety disorders at posttreatment and $77 \%$ at 3-month followup (Silverman et al., 1999). Similarly, Kendall, Hudson, Gosch, Flannery-Schroeder and Suveg (2008) reported that combined individual and family CBT resulted in $64-67 \%$ of children being without their principal diagnoses after treatment. Likewise, Hudson et al., (2008) also demonstrated that $49 \%$ of children who participated in group CBT no longer fulfilled diagnoses of any anxiety disorder after 3-month follow-up. In addition, our previous meta-analysis for CBT for children with anxiety disorders in Western countries suggested that there were few differences between individual and group therapy (Ishikawa et al., 2007). The present study also suggested that there were no differences between individual or group formats in terms of diagnostic-free rates.

Interestingly, the results also suggested that anxiety and depression significantly improved during follow-up. In addition, this beneficial effect was also found in diagnostic interviews, in that more children and adolescents no longer fulfilled criteria for an anxiety disorder at follow-up than posttreatment. Considering therapeutic characteristics of CBT for children and adolescents, it has been suggested that it is necessary to teach coping skills for daily adversities and encourage control of anxious symptoms both by the children themselves and by them in cooperation with their family members (Prins and Ollendick, 2003). We appear to have some limited support for this notion. That is, after children and adolescents applied the cognitive-behavioural skills in treatment, their psychopathological symptoms shifted towards improvement following treatment in their everyday life circumstances. In fact, the relationship between improvements in cognitive errors and internalizing psychopathologies became clearer as time passed. Of course, since no control group was used in the present study, we cannot be sure that these effects were the direct result of our treatments. However, based on previous studies that have used waitlist control groups in Western countries (Ishikawa et al., 2007), changes in the absence of active treatment are unlikely.

In the present study, not only was improvement in psychological symptoms observed but also an alteration of cognitive errors was witnessed. As pointed out by Prins and Ollendick (2003), there are few studies that examine the relations between proposed mediators of change and treatment effects. Although previous studies suggest that change in anxious self-talk predicts improvement in self-reported anxiety measures (Kendall and Treadwell, 2007; Treadwell and Kendall, 1996), these studies, like the current one, did not establish the required temporal relations between the candidate mediator and the treatment outcomes. That is, change in the cognitive mediator was not shown to change before change in the outcome measure. Still, it is noteworthy that change in cognitive errors was targeted as one of our principal therapeutic components in treatments and that a relationship between cognitive change and anxiety and depression improvement was demonstrated.

There are some obvious limitations in our study. First, this study did not use a randomized control design; such a study is desperately needed. Second, we were not able to control for therapist expectancies because our therapists were not randomly assigned to the treatment conditions. Third, since we did not use a control group, our interviewers were not blinded. All interviewers knew in which formats of CBT the youth had been involved. Fourth, except 
for the diagnostic interview, most of our outcome measures were child self-reports. Although self-report measures are useful assessment for children and adolescents with internalizing disorders, parents' and teachers' reports, as well as behavioural observations, should be used in future studies. Fifth, we failed to diagnose "taijin kyofusyo", a Japanese culture-bound syndrome, and discriminate it from other anxiety symptoms. Taijin kyofusyo is characterized by an intense fear that one's body parts or functions displease, embarrass, or are offensive to others (American Psychiatric Association, 2000). Since little is known about taijin kyofusyo in younger ages, it is important that future work evaluate the usefulness of psychotherapy developed in Western countries for cultural-bound syndromes. Finally, we were not able to audiotape or videotape our treatment sessions and, as a result, we were not able to collect data regarding treatment integrity.

Despite these limitations, the present study marks the first step toward evaluation and dissemination of CBT for children and adolescents in Asian countries. Compared with previous trials conducted in the US (e.g. Beidel et al., 2007; Ollendick et al., 2009), recruitment rates in this study were quite low. Although speculative, this might be related to the fact that most Japanese people who have experienced a severe or moderate psychological problem tend not to seek professional treatment or health care (Kawakami et al., 2005). In particular, anxiety-related problems in children and adolescents tend to be overlooked by parents and teachers because these children are viewed as compliant and "good" children under Japanese social norm (see Ishikawa, 2006; Ollendick and Ishikawa, in press). Nevertheless, the overall level of anxiety symptoms reported by Japanese children and adolescents are similar to those in Western countries (Ishikawa et al., 2009) and children with anxiety symptoms report also feeling inadequate in peer relationship and school performance (Ishikawa, Ota and Sakano, 2003). In addition, there are very few randomized control trials of psychosocial interventions in Japan even for various forms of adult psychopathology, let alone child psychopathologies. In terms of feasibility and accessibility, we determined that a quasi-experimental design was useful as the first step in evaluating the efficacy of CBT in Japan. Furthermore, quasi-experimental design studies for the treatment of youth with anxiety and phobic disorders frequently precede randomized control trials in Western countries (see Ollendick and King, 2000). These studies have laid the foundation for the current progress of evidence-based psychotherapy for children and adolescents. Nonetheless, considering our obvious limitations, we recognize that more robust studies are necessary to establish the efficacy and effectiveness of CBT for children and adolescents in Japan.

\section{Acknowledgements}

This project was supported by a grant from the Ministry of Health, Labour and Welfare, Japan. We thank the research participants and their families who made this study possible. We are also grateful to Hitomi Ninomiya, Yuuko Hara, Akiko Motomura, Chiaki Takemoto, Chikako Mori and Hitoshi Tonotani for their assistance throughout this project. Finally, we would like to thank Jane Gillham, at Swarthmore College, for her helpful comments on this manuscript.

\section{References}

American Psychiatric Association (2000). Diagnostic and Statistical Manual of Mental Disorders (4th ed., text rev). Washington, DC: Author. 
Albano, A. M., Chorpita, B. F. and Barlow, D. H. (2003). Childhood anxiety disorders. In E. J. Mash and R. A. Barkley (Eds.), Childhood Psychopathology (2nd ed.). New York: Guilford Press.

Barrett, P. M. (1998). Evaluation of cognitive-behavioral group treatment for childhood anxiety disorders. Journal of Clinical Child Psychology, 27, 459-468.

Beidel, D. C., Turner, S. M., Sallee, F. R., Ammerman, R. T., Crosby, L. A. and Pathak, S. (2007). SET-C versus fluoxetine in the treatment of childhood social phobia. Journal of the American Academy of Child and Adolescent Psychiatry, 46, 1622-1632.

Benjamin, R. S., Costello, E. J. and Warren, M. (1990). Anxiety disorders in a pediatric sample. Journal of Anxiety Disorders, 4, 293-316.

Birleson, P. (1981). The validity of depressive disorder in childhood and the development of self-rating scale. Journal of Child Psychology and Psychiatry, 22, 73-88.

Burlingame, G. M., McClendon, D. T. and Alonso, J. (2011). Cohesion in group therapy. Psychotherapy, 48, 34-42.

Chen, J., Nakano, Y., Ietzugu, Ogawa, S., Funayama, T., Watanabe, N., et al. (2007). Group cognitive behavior therapy for Japanese patients with social anxiety disorder: preliminary outcome and their predictors. BMC Psychiatry, 7, 69.

Costello, E. J., Egger, H. L. and Angold, A. (2004). Developmental epidemiology of anxiety disorders. In T. H. Ollendick and J. S. March (Eds.), Phobic and Anxiety Disorders in Children and Adolescents: a clinician's guide to effective psychosocial and pharmacological intervention New York: Oxford University Press.

Essau, C. A., Sakano, Y., Ishikawa, S. and Sasagawa, S. (2004). Anxiety symptoms in Japanese and in German children. Behaviour Research and Therapy, 42, 601-612.

Hudson, J. L., Rapee, R. M., Deveney, C., Schniering, C. A., Lyneham, H. J. and Bovopoulos, N. (2008). Cognitive-behavioral treatment versus an active control for children and adolescents with anxiety disorders: a randomized trial. Journal of the American Academy of Child and Adolescent Psychiatry, 48, 533-544.

Ishikawa, S. (2006). Kodomo no fuan-sho-gai [Anxiety disorders in children]. In Y. Sakano, Y. Tanno and Y. Sugiura (Eds.), Fuan-sho-gai no rinsho-shinri-gaku [Clinical Psychology of Anxiety Disorders] (pp. 135-151). Tokyo: University of Tokyo Press.

Ishikawa, S. (in press). Cognitive errors, anxiety, and depression in Japanese children and adolescents. International Journal of Cognitive Therapy.

Ishikawa, S., Okajima, I., Matsuoka, H. and Sakano, Y. (2007). Cognitive behavioural therapy for anxiety disorders in children and adolescents: a meta-analysis. Child and Adolescent Mental Health, 12, 164-172.

Ishikawa, S., Ota, R. and Sakano, Y. (2003). The relationship between anxiety disorders tendencies and subjective school maladjustment in childhood. Japanese Journal of Counseling Science, 36, 264271.

Ishikawa, S. and Sakano, Y. (2003). Cognitive error and trait anxiety in children: development of a Children's Cognitive Error Scale. Japanese Journal of Behavior Therapy, 29, 145-157.

Ishikawa, S., Sato, H. and Sasagawa, S. (2009). Anxiety disorder symptoms in Japanese children and adolescents. Journal of Anxiety Disorders, 23, 104-111.

Ishikawa, S., Shimotsu, S. and Sato, Y. (2008). Short-term and group cognitive behavior therapy for children with anxiety disorders. Japanese Journal of Psychiatric Treatment, 23, 14811490 .

Kawakami, N., Takeshima, T., Ono, Y., Uda, H., Hata, Y., Nakane, Y., et al. (2005). Twelvemonth prevalence, severity, and treatment of common mental disorders in communities in Japan: preliminary finding from the World Mental Health Japan Survey 2002-2003. Psychiatry and Clinical Neurosciences, 59, 441-452.

Kendall, P. C. (1994). Treating anxiety disorders in children: results of randomized clinical trial. Journal of Consulting and Clinical Psychology, 62, 100-110. 
Kendall, P. C., Hudson, J. L., Gosch, E., Flannery-Schroeder, E. and Suveg, C. (2008). Cognitivebehavioral therapy for anxiety disordered youth: a randomized clinical trial evaluating child and family modalities. Journal of Consulting and Clinical Psychology, 76, 282-297.

Kendall, P. C., Safford, S., Flannery-Schroeder, E. C. and Webb, A. (2004). Child anxiety treatments: outcomes in adolescence and impact on substance use and depression at 7.4 year follow-up. Journal of Consulting and Clinical Psychology, 72, 276-287.

Kendall, P. C. and Treadwell, K. R. H. (2007). The role of self-statements as a mediator in treatment for youth with anxiety disorders. Journal of Consulting and Clinical Psychology, 75, 869-880.

Leitenberg, H., Yost, L. W. and Carroll-Wilson, M. (1986). Negative cognitive errors in children questionnaire development, normative data, and comparisons between children with and without selfreported symptoms of depression, low self-esteem, and evaluation anxiety. Journal of Consulting and Clinical Psychology, 54, 528-536.

Markus, H. R. and Kitayama, S. (1991). Culture and the self: implications for cognition, emotion, and motivation. Psychological Bulletin, 98, 224-253.

Murata, T., Shimizu, A., Mori, Y. and Oushima, S. (1996). Childhood depressive state in the school situation: consideration from the Birleson's scale. Saishin Seishin Igaku, 1, 131-138.

Ollendick, T. H. and Ishikawa, S. (in press). Interpersonal and social factors in the treatment of child and adolescent anxiety disorders. In C. A. Essau and T. H. Ollendick (Eds.), Treatment of Anxiety Disorders in Children and Adolescents. Chichester: Wiley-Blackwell.

Ollendick, T. H. and King, N. J. (1994). Assessment and treatment of internalizing problems: the role of longitudinal data. Journal of Consulting and Clinical Psychology, 62, 918-927.

Ollendick, T. H. and King, N. J. (1998). Empirically supported treatments for children with phobic and anxious disorders: current status. Journal of Clinical Child Psychology, 27, 156-167.

Ollendick, T. H. and King, N. J. (2000). Empirically supported treatments for children and adolescents. In P. C. Kendall (Ed), Child and Adolescent Therapy: cognitive-behavioral procedures (2nd ed.). New York: Guilford Press.

Ollendick, T. H. and March, J. S. (2004). Phobic and Anxiety Disorders: a clinician's guide to effective psychosocial and pharmacological interventions. New York: Oxford University Press.

Ollendick, T. H., Öst, L-H., Reuterskiöld, L., Costa, N., Cederlund, R., Sirbu, C., et al. (2009). One-session treatment of specific phobias in youth: a randomized clinical trial in the United States and Sweden. Journal of Clinical Child and Adolescents Psychology, 77, 504-516.

Pine, D. S., Cohen, P., Gurley, D., Brook, J. and Ma, Y. (1998). The risk for early-adulthood anxiety and depressive disorders in adolescents with anxiety and depressive disorders. Archive General Psychiatry, 55, 56-64.

Prins, P. J. M. and Ollendick, T. H (2003). Cognitive change and enhanced coping: missing mediational links in cognitive behavior therapy with anxiety-disordered children. Clinical Child and Family Psychology Review, 6, 87-105.

Rohde, P., Lewinsohn, P. M. and Seeley, J. R. (1991). Comorbidity of unipolar depression: II. comorbidity with other mental disorders in adolescents and adults. Journal of Abnormal Psychology, 100, 214-222.

Rohde, P., Lewinsohn, P. M. and Seeley, J. R. (1996). Psychiatric comorbidity with problematic alcohol use in high school adolescents. Journal of the American Academy of Child and Adolescent Psychiatry, 35, 101-109.

Sato, H., Ishikawa, S. and Arai, K. (2004). The effect of logical thinking error on the symptoms of depression and anxiety disorders in children. Japanese Journal of Behavioral Medicine, 10, 73-80.

Shimotsu, S., Nagao, A., Emura, R., Ogata, A., Ishikawa, S., Hiejima, S., et al. (2011). Effectiveness of group cognitive behavior therapy in a community clinic. Japanese Journal of Cognitive Therapy, $4,76-85$.

Silverman, W. K. and Albano, A. M. (1996). Manual for Anxiety Disorders Interview Schedule for DSM-IV: child and parent versions. San Antonio, TX: Graywind Publications. 
Silverman, W. K., Kurtines, W. M., Ginsburg, G. S., Weems, C. F., Lumpkin, P. W. and Carmichael, D. H. (1999). Treating anxiety disorders in children with group cognitive-behavioral therapy: a randomized clinical trial. Journal of Consulting and Clinical Psychology, 67, 995-1003.

Silverman, W. K., Pina, A. A. and Viswesvaran, C. (2008). Evidence-based psychosocial treatments for phobic and anxiety disorders in children and adolescents. Journal of Clinical Child and Adolescents Psychology, 37, 105-130.

Spence, S. H. (1998). A measure of anxiety symptoms among children. Behaviour Research and Therapy, 36, 545-566.

Treadwell, K. R. H. and Kendall, P. C. (1996). Self-talk in youth with anxiety disorders: states of mind, content specificity, and treatment outcome. Journal of Consulting and Clinical Psychology, 64, 941-950.

Walkup, J. T., Albano, A. M., Piacentini, J., Birmaher, B., Compton, S. N., Sherrill, J. T., et al. (2008). Cognitive behavioral therapy, sertraline, or a combination in childhood anxiety. New England Journal of Medicine, 359, 2753-2766. 